\title{
Efficient Computation of the Pareto Boundary for the Two-User MISO Interference Channel with Multi-User Decoding Capable Receivers
}

\author{
Johannes Lindblom, Eleftherios Karipidis and Erik G. Larsson
}

\section{Linköping University Post Print}

N.B.: When citing this work, cite the original article.

(C2011 IEEE. Personal use of this material is permitted. However, permission to reprint/republish this material for advertising or promotional purposes or for creating new collective works for resale or redistribution to servers or lists, or to reuse any copyrighted component of this work in other works must be obtained from the IEEE.

Johannes Lindblom, Eleftherios Karipidis and Erik G. Larsson, Efficient Computation of the Pareto Boundary for the Two-User MISO Interference Channel with Multi-User Decoding Capable Receivers, 2011, Proceedings of the 4th IEEE International Workshop on Computational Advances in Multi-Sensor Adaptive Processing (CAMSAP), 241-244. 


\title{
Efficient Computation of the Pareto Boundary for the Two-User MISO Interference Channel with Multi-User Decoding Capable Receivers
}

\author{
Johannes Lindblom, Eleftherios Karipidis, and Erik G. Larsson \\ Communication Systems Division, Department of Electrical Engineering (ISY), Linköping University \\ SE-581 83 Linköping, Sweden. \{lindblom,karipidis, erik.larsson\}@isy.liu.se
}

\begin{abstract}
We study the two-user multiple-input single-output (MISO) interference channel for the scenario where the transmitters have perfect channel state information and employ singlestream beamforming. We assume that the receivers are able of decoding the data from both transmitters. Hence, the signal from the interfering transmitter might be decoded, treating the desired signal as noise, and subtracted from the received signal. We propose an efficient method for finding the Pareto boundary of the corresponding achievable rate region. This method has a complexity which is constant in the number of transmit antennas.
\end{abstract}

\section{INTRODUCTION}

We study a wireless system where two transmitter (TX) - receiver (RX) pairs, or links, operate simultaneously in the same frequency band. Hence, the links interfere with each other. This situation can be modeled via the so-called interference channel (IC) [1]. The TXs employ $n_{T} \geq 2$ antennas each, whereas the RXs are equipped with a single antenna each. Hence, the system is a multiple-input singleoutput (MISO) IC [2]. When the RXs treat the interference as noise, see e.g. [2]-[4], the interference can substantially degrade the performance of the two links.

The capacity region of the IC is unknown. However, we know that for strong interference, it is optimal to first decode the interference treating the desired signal as noise, subtract the interference, and then decode the desired message [5]. For weak interference, it is optimal to treat the interference as additive noise [6]. Motivated by these facts, we assume that the RXs are capable to decode the interference and subtract it from the received signal before decoding the intended data. Because of practical reasons, the RXs do the decoding independently. Moreover, we assume that both TXs have perfect channel state information and use Gaussian coding with single-stream beamforming. Given these assumptions, we obtain an achievable rate region. Herein, the focus is to efficiently find the so-called Pareto boundary of this region. The Pareto boundary consists of the points where we cannot increase the rate of one link without decreasing the rate of the other.

The MISO IC with multi-user decoding (MUD) capable RXs was first investigated in [2], where the authors illustrated

This work has been supported in part by the Swedish Research Council (VR), the Swedish Foundation of Strategic Research (SSF), and the Excellence Center at Linköping-Lund in Information Technology (ELLIIT). This work has been performed in the framework of the European research project SAPHYRE, which is partly funded by the European Union under its FP7 ICT Objective 1.1 - The Network of the Future. E. G. Larsson is a Royal Swedish Academy of Sciences (KVA) Research Fellow supported by a grant from the Knut and Alice Wallenberg Foundation. the potential gain of MUD compared to single-use decoding. In [7], an achievable rate region for the described scenario was defined. The authors of [7] proposed a parameterization of the beamforming vectors that achieve Pareto-optimal (PO) rate points. This parameterization does only yield necessary conditions that the beamforming vectors have to separately fulfill. That is, we only get pairs of beamforming vectors which potentially give PO operating points. In order to find the Pareto boundary, we have to perform a brute-force search over all rate pairs. However, the parameterization gives us some insight. When the RXs treat interference as noise, the PO beamforming vectors are obtained by trading off between maximizing the own rate and avoid creating interference. On the other hand, when the RXs decode the interference, we have a trade-off between maximizing the own rate and causing extra interference in order to aid the decoding of the interference.

Contributions: We propose a method that jointly finds a pair of beamforming vectors that yield an arbitrary PO point. We find the Pareto boundary in two steps. First, we compute the boundaries corresponding to the four scenarios of 1) both RXs decode the interference, 2) both RXs treat the interference as additive noise, 3) $\mathrm{RX}_{1}$ decodes the interference while $\mathrm{RX}_{2}$ treats it as noise, and 4) $\mathrm{RX}_{1}$ treats the interference as noise while $\mathrm{RX}_{2}$ decodes it. Second, the rate region for the MISO IC with MUD is obtained as the union of these four regions.

Notation: $\boldsymbol{\Pi}_{\boldsymbol{x}} \triangleq \boldsymbol{x} \boldsymbol{x}^{H} /\|\boldsymbol{x}\|^{2}$ is the orthogonal projection onto the vector $\boldsymbol{x}$, whereas $\boldsymbol{\Pi}_{\boldsymbol{x}}^{\perp} \triangleq \boldsymbol{I}-\boldsymbol{\Pi}_{\boldsymbol{x}}$ is the orthogonal projection onto the orthogonal complement of $x$. By $x \sim$ $\mathcal{C N}\left(0, \sigma^{2}\right)$ we say that $x$ is a zero-mean complex circularlysymmetric Gaussian random variable with variance $\sigma^{2}$.

\section{SySTEM MODEL}

We assume that the transmissions consist of scalar coding followed by beamforming and that all propagation channels are frequency-flat. The matched-filtered symbol-sampled complex baseband data received by $\mathrm{RX}_{1}$ is modeled as ${ }^{1}$

$$
y_{1}=\boldsymbol{h}_{11}^{H} \boldsymbol{w}_{1} s_{1}+\boldsymbol{h}_{21}^{H} \boldsymbol{w}_{2} s_{2}+e_{1} .
$$

In (1), $\boldsymbol{h}_{11}, \boldsymbol{h}_{21} \in \mathbb{C}^{n_{T}}$, are the (conjugated) channel vectors for the links $\mathrm{TX}_{1} \rightarrow \mathrm{RX}_{1}$ and $\mathrm{TX}_{2} \rightarrow \mathrm{RX}_{1}$, respectively. We assume that the channels are perfectly known at the TXs. Also, $\boldsymbol{w}_{1}, \boldsymbol{w}_{2} \in \mathbb{C}^{n_{T}}$ are the beamforming vectors employed by $\mathrm{TX}_{1}$

\footnotetext{
${ }^{1}$ Whenever an expression is introduced only with respect to link 1 , the expression for link 2 is obtained by interchanging the indices.
} 
and $\mathrm{TX}_{2}$, respectively, $s_{1}, s_{2} \sim \mathcal{C N}(0,1)$ are the transmitted symbols of $\mathrm{TX}_{1}$ and $\mathrm{TX}_{2}$, respectively, and $e_{1} \sim \mathcal{C N}\left(0, \sigma^{2}\right)$ models the receiver noise at $\mathrm{RX}_{1}$.

The achievable rates depend on the received power. Specifically, for $\mathrm{RX}_{1}$ we define $p_{1}\left(\boldsymbol{w}_{1}\right) \triangleq\left|\boldsymbol{h}_{11}^{H} \boldsymbol{w}_{1}\right|$ to be the power received from $\mathrm{TX}_{1}$ over the direct channel and $q_{1}\left(\boldsymbol{w}_{2}\right) \triangleq$ $\left|\boldsymbol{h}_{21}^{H} \boldsymbol{w}_{2}\right|$ to be the power received from $\mathrm{TX}_{2}$ over the cross-talk channel. There is a power constraint that we, without loss of generality, set to 1 and define the set of feasible beamforming vectors as $\mathcal{W} \triangleq\left\{\boldsymbol{w} \in \mathbb{C}^{n_{T}} \mid\|\boldsymbol{w}\|^{2} \leq 1\right\}$.

\section{An Achievable Rate Region}

In this section, we construct an achievable rate region for the described scenario. Each pair of beamforming vectors $\left(\boldsymbol{w}_{1}, \boldsymbol{w}_{2}\right)$ and combination of decoding strategies (decode the interference $(d)$ or treat it as noise $(n)$ ) is associated with maximum achievable rates. We denote these rates, in bits per channel use (bpcu), $R_{i}^{x y}\left(\boldsymbol{w}_{1}, \boldsymbol{w}_{2}\right), i=1,2$, where $x$ and $y$ stand for the decoding strategies $n$ or $d$. For each decoding strategy, we obtain a rate region by taking the union over all feasible beamforming vectors, i.e.

$$
\mathcal{R}^{x y} \triangleq \bigcup_{\left(\boldsymbol{w}_{1}, \boldsymbol{w}_{2}\right) \in \mathcal{W}^{2}}\left(R_{1}^{x y}\left(\boldsymbol{w}_{1}, \boldsymbol{w}_{2}\right), R_{2}^{x y}\left(\boldsymbol{w}_{1}, \boldsymbol{w}_{2}\right)\right),
$$

where $x$ and $y$ stand for the decoding strategies $n$ or $d$. The maximum achievable rate for each pair of beamforming vectors $\left(\boldsymbol{w}_{1}, \boldsymbol{w}_{2}\right)$ is as follows [7]:

Both RXs treat the interference as noise: When both RXs treat the interference as noise, link 1 achieves the rate [2]

$$
R_{1}^{n n}\left(\boldsymbol{w}_{1}, \boldsymbol{w}_{2}\right)=\log _{2}\left(1+p_{1}\left(\boldsymbol{w}_{1}\right) /\left(q_{1}\left(\boldsymbol{w}_{2}\right)+\sigma^{2}\right)\right) .
$$

$\mathbf{R X}_{1}$ decodes the interference, $\mathbf{R X}_{2}$ treats it as additive noise: Since $\mathrm{RX}_{1}$ decodes and subtracts the interference caused by $\mathrm{TX}_{2}$, it experiences an interference-free signal and achieves the rate

$$
R_{1}^{d n}\left(\boldsymbol{w}_{1}\right)=\log _{2}\left(1+p_{1}\left(\boldsymbol{w}_{1}\right) / \sigma^{2}\right) .
$$

$\mathrm{RX}_{1}$ will be able to decode interference from $\mathrm{TX}_{2}$ if the rate of link 2 satisfies

$$
R_{2} \leq \log _{2}\left(1+q_{1}\left(\boldsymbol{w}_{2}\right) /\left(p_{1}\left(\boldsymbol{w}_{1}\right)+\sigma^{2}\right)\right) .
$$

Since $\mathrm{RX}_{2}$ does not decode the interference, the rate of link 2 must also satisfy

$$
R_{2} \leq \log _{2}\left(1+p_{2}\left(\boldsymbol{w}_{2}\right) /\left(q_{2}\left(\boldsymbol{w}_{1}\right)+\sigma^{2}\right)\right) .
$$

The maximum achievable rate $R_{2}^{d n}\left(\boldsymbol{w}_{1}, \boldsymbol{w}_{2}\right)$ is the minimum of the right-hand sides of (5) and (6). For link 2, we note that the maximum achievable rate does not necessarily exploit the signal-to-interference-plus-noise (SINR) ratio at $\mathrm{RX}_{2}$ to the full extent. Actually, it might hold back on rate to facilitate $\mathrm{RX}_{1}$ to decode the signal of link 2. This fact was not exploited in [2], where the description leads to too restrictive conditions.

$\mathbf{R X}_{2}$ decodes the interference, $\mathbf{R X}_{1}$ treats it as additive noise: This case is identical to $\mathcal{R}^{n d}$, but with interchanged indices.
Both RXs decode the interference: Both RXs decode the interference before decoding their desired signals. When $\mathrm{RX}_{1}$ has decoded the interference from $\mathrm{TX}_{2}$, the desired signal can be decoded if the rate of link 1 satisfies

$$
R_{1} \leq \log _{2}\left(1+p_{1}\left(\boldsymbol{w}_{1}\right) / \sigma^{2}\right) .
$$

$\mathrm{RX}_{2}$ can decode the interference caused by $\mathrm{TX}_{1}$ if the rate of link 1 satisfies

$$
R_{1} \leq \log _{2}\left(1+q_{2}\left(\boldsymbol{w}_{1}\right) /\left(p_{2}\left(\boldsymbol{w}_{2}\right)+\sigma^{2}\right)\right)
$$

So, the maximum achievable rate of link $1, R_{1}^{d d}\left(\boldsymbol{w}_{1}, \boldsymbol{w}_{2}\right)$, is the minimum of the right-hand sides of (7) and (8).

The achievable rate region: The rate region for the MISO IC with MUD capability is obtained as

$$
\mathcal{R}=\mathcal{R}^{n n} \cup \mathcal{R}^{d n} \cup \mathcal{R}^{n d} \cup \mathcal{R}^{d d} .
$$

We are interested in finding the so-called Pareto boundary of the region $\mathcal{R}$. The Pareto boundary consists of PO points, where Pareto-optimality is defined as follows.

Definition 1. A rate pair $\left(R_{1}^{\star}, R_{2}^{\star}\right) \in \mathcal{R}$ is Pareto-optimal if there is no other rate pair $\left(R_{1}, R_{2}\right) \in \mathcal{R}$ with $\left(R_{1}, R_{2}\right)>$ $\left(R_{1}^{\star}, R_{2}^{\star}\right)$. (The inequality is component-wise.)

Note that Def. 1 also includes the horizontal and vertical sections of the Pareto boundary. Hence the definition defines weak Pareto-optimality. Graphically, the Pareto boundary is the north-east boundary of the region. To find the Pareto boundary of $\mathcal{R}$, we first find the Pareto boundaries of $\mathcal{R}^{n n}$, $\mathcal{R}^{d n}, \mathcal{R}^{n d}$, and $\mathcal{R}^{d d}$. Second, we consider as boundary of $\mathcal{R}$ the boundary of the union of $\mathcal{R}^{n n}, \mathcal{R}^{d n}, \mathcal{R}^{n d}$, and $\mathcal{R}^{d d}$. For each of these regions, we do as follows. Let $\left(R_{1}^{\star}, R_{2}^{\star}\right)$ be an arbitrary point on the Pareto boundary. In order to find this point, we fix the rate of link 1 at $R_{1}^{\star}$ and maximize $R_{2}$ in order to get $R_{2}^{\star}$. Due to the monotonicity of the logarithmic function, we formulate a SINR optimization problem. We define $\gamma_{i}^{x y}\left(\boldsymbol{w}_{1}, \boldsymbol{w}_{2}\right) \triangleq 2^{R_{i}^{x y}\left(\boldsymbol{w}_{1}, \boldsymbol{w}_{2}\right)}-1$ to be the SINR needed to achieve $R_{i}^{x y}\left(\boldsymbol{w}_{1}, \boldsymbol{w}_{2}\right)$. The problem of finding the optimal $\gamma_{2}^{\star}$ for a given $\gamma_{1}^{\star}$ can be formulated as [3]

$$
\begin{aligned}
& \underset{\left(\boldsymbol{w}_{1}, \boldsymbol{w}_{2}\right) \in \mathcal{W}^{2}}{\operatorname{maximize}} \gamma_{2}^{x y}\left(\boldsymbol{w}_{1}, \boldsymbol{w}_{2}\right), \\
& \text { subject to } \gamma_{1}^{x y}\left(\boldsymbol{w}_{1}, \boldsymbol{w}_{2}\right)=\gamma_{1}^{\star} .
\end{aligned}
$$

The optimal solution of (10)-(11) is the pair of beamforming vectors $\left(\boldsymbol{w}_{1}^{\star}, \boldsymbol{w}_{2}^{\star}\right)$ enabling $\left(R_{1}^{\star}, R_{2}^{\star}\right)$.

\section{EfFicient Computation of the Pareto Boundary}

In this section, we propose efficient methods for finding the boundaries of $\mathcal{R}^{n n}, \mathcal{R}^{d n}, \mathcal{R}^{n d}$, and $\mathcal{R}^{d d}$. The focus is on $\mathcal{R}^{d n}$ and $\mathcal{R}^{d d}$. Due to symmetry, the problem of computing the boundary of $\mathcal{R}^{\text {nd }}$ is identical to that of finding the boundary of $\mathcal{R}^{d n}$. For $\mathcal{R}^{n n}$, we have previously proposed two methods. In [3] we computed an arbitrary point on the boundary via a sequence of second-order cone (SOC) programs. In [4], we gave a closed-form parameterization of the beamforming vectors that yield $\mathrm{PO}$ rate points. The methods for finding $\mathcal{R}^{d n}, \mathcal{R}^{n d}$, and $\mathcal{R}^{d d}$ devised in the sequel are novel. 


\section{A. Only One RX Decodes Interference}

Here, we consider the boundary of the region $\mathcal{R}^{d n}$, i.e. the region consisting of the points where $\mathrm{RX}_{1}$ is able to decode the interference while $\mathrm{RX}_{2}$ treats it as noise. We insert (4)-(6) in (10)-(11) and obtain the resulting problem

$$
\begin{array}{ll}
\underset{\gamma_{2} \in \mathbb{R}_{+},\left(\boldsymbol{w}_{1}, \boldsymbol{w}_{2}\right) \in \mathcal{W}^{2}}{\operatorname{maximiz}} & \gamma_{2} \\
\text { subject to } & p_{1}\left(\boldsymbol{w}_{1}\right) / \sigma^{2}=\gamma_{1}^{\star}, \\
& q_{1}\left(\boldsymbol{w}_{2}\right) /\left(p_{1}\left(\boldsymbol{w}_{1}\right)+\sigma^{2}\right) \geq \gamma_{2}, \\
& p_{2}\left(\boldsymbol{w}_{2}\right) /\left(q_{2}\left(\boldsymbol{w}_{1}\right)+\sigma^{2}\right) \geq \gamma_{2} .
\end{array}
$$

This is nonconvex, because (13) is a quadratic equality and (14), (15) are nonconvex quadratic inequalities parameterized by $\gamma_{2}$. However, in [7] it was shown that the beamforming vectors that solve (12)-(15) can be parameterized as

$$
\begin{aligned}
& \boldsymbol{w}_{1}=x_{1} \frac{\boldsymbol{\Pi}_{\boldsymbol{h}_{12}} \boldsymbol{h}_{11}}{\left\|\boldsymbol{\Pi}_{\boldsymbol{h}_{12}} \boldsymbol{h}_{11}\right\|}+y_{1} \frac{\boldsymbol{\Pi}_{\boldsymbol{h}_{12}}^{\perp} \boldsymbol{h}_{11}}{\left\|\boldsymbol{\Pi}_{\boldsymbol{h}_{12}}^{\perp} \boldsymbol{h}_{11}\right\|}, \\
& \boldsymbol{w}_{2}=x_{2} \frac{\boldsymbol{\Pi}_{\boldsymbol{h}_{22}} \boldsymbol{h}_{21}}{\left\|\boldsymbol{\Pi}_{\boldsymbol{h}_{22}} \boldsymbol{h}_{21}\right\|}+y_{2} \frac{\boldsymbol{\Pi}_{\boldsymbol{h}_{22}}^{\perp} \boldsymbol{h}_{21}}{\left\|\boldsymbol{\Pi}_{\boldsymbol{h}_{22}}^{\perp} \boldsymbol{h}_{21}\right\|},
\end{aligned}
$$

where $\left(x_{i}, y_{i}\right) \in \mathcal{Q} \triangleq\left\{(x, y) \mid x, y \geq 0, x^{2}+y^{2} \leq 1\right\}$. We see that $\mathcal{Q}$ is a quarter disk, which is a convex set. Using the parameterization (16)-(17), we propose a closed-form solution of (12)-(15). By using the parameterization (16)-(17), we note that $y_{1}$ does not affect $q_{2}\left(\boldsymbol{w}_{1}\right)$ and $y_{2}$ does not affect $p_{2}\left(\boldsymbol{w}_{2}\right)$. Hence, we can ignore the complex phases of $\boldsymbol{h}_{12}^{H} \boldsymbol{w}_{1}$ and $\boldsymbol{h}_{22}^{H} \boldsymbol{w}_{2}$. Also, we see that the inner products $\boldsymbol{h}_{11}^{H} \boldsymbol{w}_{1}$ and $\boldsymbol{h}_{21}^{H} \boldsymbol{w}_{2}$ are real and positive. Then, we define $t \triangleq \sqrt{\gamma_{2}}$, insert (13) in (14) and equivalently write (12)-(17) as

$$
\begin{array}{ll}
\underset{t \in \mathbb{R}_{+},\left(x_{i}, y_{i}\right) \in \mathcal{Q}, i=1,2}{\operatorname{maximize}} & t \\
\text { subject to } & \alpha x_{1}+\tilde{\alpha} y_{1}=\sqrt{\gamma_{1}^{\star} \sigma^{2}}, \\
& \beta_{2} x_{2}+\tilde{\beta}_{2} y_{2} / \sqrt{\left(\gamma_{1}^{\star}+1\right) \sigma^{2}} \geq t, \\
& g_{22} x_{2} / \sqrt{g_{12}^{2} x_{1}^{2}+\sigma^{2}} \geq t .
\end{array}
$$

The coefficients in (18)-(21) are defined in Tab. I. We solve (18)-(21) in two steps. First, we solve for $\left(x_{1}, y_{1}\right)$ and we call the optimal solution $\left(x_{1}^{\star}, y_{1}^{\star}\right)$. We note that $x_{1}$ and $y_{1}$ only appear in constraints (19) and (21). We make the left-hand side of (21) as large as possible by minimizing $x_{1}$ subject to the constraint (19):

$$
\begin{array}{ll}
\underset{\left(x_{1}, y_{1}\right) \in \mathcal{Q}}{\operatorname{minimize}} & x_{1} \\
\text { subject to } & \alpha x_{1}+\tilde{\alpha} y_{1}=\sqrt{\gamma_{1}^{\star} \sigma^{2}} .
\end{array}
$$

Second, we insert the optimal solution $x_{1}^{\star}, y_{1}^{\star}$ of (22)-(23) into (18)-(21) and obtain

$$
\begin{array}{ll}
\underset{t \in \mathbb{R}_{+},\left(x_{2}, y_{2}\right) \in \mathcal{Q}}{\operatorname{maximize}} & t \\
\text { subject to } & \beta_{2} x_{2}+\tilde{\beta}_{2} y_{2} / \sqrt{\left(\gamma_{1}^{\star}+1\right) \sigma^{2}} \geq t, \\
& g_{22} x_{2} / \sqrt{g_{12}^{2}\left(x_{1}^{\star}\right)^{2}+\sigma^{2}} \geq t .
\end{array}
$$

$$
\begin{array}{|ll|}
\alpha \triangleq\left\|\boldsymbol{\Pi}_{\boldsymbol{h}_{12}} \boldsymbol{h}_{11}\right\|, & \tilde{\alpha} \triangleq\left\|\boldsymbol{\Pi}_{\boldsymbol{h}_{12}}^{\perp} \boldsymbol{h}_{11}\right\| \\
\beta_{1} \triangleq\left\|\boldsymbol{\Pi}_{\boldsymbol{h}_{11}} \boldsymbol{h}_{12}\right\|, & \tilde{\beta}_{1} \triangleq\left\|\boldsymbol{\Pi}_{\boldsymbol{h}_{11}}^{\perp} \boldsymbol{h}_{12}\right\| \\
\beta_{2} \triangleq\left\|\boldsymbol{\Pi}_{\boldsymbol{h}_{22}} \boldsymbol{h}_{21}\right\|, & \tilde{\beta}_{2} \triangleq\left\|\boldsymbol{\Pi}_{\boldsymbol{h}_{22}}^{\perp} \boldsymbol{h}_{21}\right\| \\
g_{i j} \triangleq\left\|\boldsymbol{h}_{i j}\right\|, & i, j=1,2 \\
\hline
\end{array}
$$

TABLE I

DEFINITION OF CONSTANTS.

The solutions of (22)-(23) and (24)-(26) are summarized in the following proposition.

Proposition 1. The optimal solution $\left(x_{1}^{\star}, y_{1}^{\star}\right)$ of (22)-(23) is

$$
\begin{aligned}
& x_{1}^{\star}=\max \left\{0, \frac{1}{g_{11}^{2}}\left(\alpha \sqrt{\gamma_{1}^{\star} \sigma^{2}}-\tilde{\alpha} \sqrt{g_{11}^{2}-\gamma_{1}^{\star} \sigma^{2}}\right)\right\}, \\
& y_{1}^{\star}= \begin{cases}\sqrt{\gamma_{1}^{\star} \sigma^{2}} / \tilde{\alpha}, & x_{1}^{\star}=0, \\
\sqrt{1-\left(x_{1}^{\star}\right)^{2}}, & \text { otherwise. }\end{cases}
\end{aligned}
$$

Then, the optimal value of (24)-(26) is given as

$$
\gamma_{2}{ }^{\star}= \begin{cases}g_{22}^{2} /\left(\left(x_{1}^{\star} g_{12}\right)^{2}+\sigma^{2}\right), & a \leq b, \\ g_{21}^{2} /\left(\sigma^{2}\left(\gamma_{1}^{\star}+1\right)\right), & a b>b^{2}+c^{2} \\ \frac{g_{22}^{2} c^{2}}{\left(\left(x_{1}^{\star} g_{12}\right)^{2}+\sigma^{2}\right)\left((a-b)^{2}+c^{2}\right)}, & \text { otherwise, }\end{cases}
$$

for

$$
\begin{aligned}
& x_{2}^{\star}= \begin{cases}1, & a \leq b, \\
b / \sqrt{b^{2}+c^{2}}, & a b>b^{2}+c^{2} \\
c / \sqrt{c^{2}+(a-b)^{2}}, & \text { otherwise, }\end{cases} \\
& y_{2}^{\star}=\sqrt{1-\left(x_{2}^{\star}\right)^{2}}
\end{aligned}
$$

where

$$
\left\{\begin{array}{l}
a \triangleq g_{22} / \sqrt{\left(x_{1}^{\star}\right)^{2} g_{12}^{2}+\sigma^{2}} \\
b \triangleq \beta_{2} / \sqrt{\sigma^{2}\left(\gamma_{1}^{\star}+1\right)} \\
c \triangleq \tilde{\beta}_{2} / \sqrt{\sigma^{2}\left(\gamma_{1}^{\star}+1\right)}
\end{array}\right.
$$

The optimal $\left(\boldsymbol{w}_{1}^{\star}, \boldsymbol{w}_{2}^{\star}\right)$ is obtained by inserting (27)-(28) and (30)-(31) into (16)-(17).

Prop. 1 provides a scheme for evaluating the Pareto boundary quickly and exactly, by providing $\gamma_{2}^{\star}$ as an explicit function of $\gamma_{1}^{\star}$, in closed-form. In order to find the entire boundary, we vary $\gamma_{1}^{\star}$ over the interval $\left[0, g_{11}^{2} / \sigma^{2}\right]$. Note that the upper bound, $g_{11}^{2} / \sigma^{2}$, is the largest value that $p_{1}\left(\boldsymbol{w}_{1}\right) / \sigma^{2}$ can assume when $\boldsymbol{w}_{1} \in \mathcal{W}$ and corresponds to the rightmost segment of the Pareto boundary. We note that once the constants in Tab. I are computed, the complexity is constant with respect to the number of transmit antennas. From Prop. 1 we note that $\mathrm{TX}_{2}$ will always use full power at the Pareto boundary, whereas $\mathrm{TX}_{1}$ might use less than full power. This was proven in [7].

\section{B. Both RXs Decode Interference}

Here we consider the boundary of the regions $\mathcal{R}^{d d}$, i.e. the region consisting of the points where both $\mathrm{RX}_{1}$ and $\mathrm{RX}_{2}$ decode the interference. We insert (7)-(8) in (10)-(11) and obtain the resulting problem 
$\underset{\gamma_{2} \in \mathbb{R}_{+},\left(\boldsymbol{w}_{1}, \boldsymbol{w}_{2}\right) \in \mathcal{W}^{2}}{\operatorname{maximize}} \gamma_{2}$

subject to

$$
\begin{aligned}
& p_{1}\left(\boldsymbol{w}_{1}\right) / \sigma^{2} \geq \gamma_{1}^{\star}, \\
& q_{2}\left(\boldsymbol{w}_{1}\right) /\left(p_{2}\left(\boldsymbol{w}_{2}\right)+\sigma^{2}\right) \geq \gamma_{1}^{\star}, \\
& p_{2}\left(\boldsymbol{w}_{2}\right) / \sigma^{2} \geq \gamma_{2}, \\
& q_{1}\left(\boldsymbol{w}_{2}\right) /\left(p_{1}\left(\boldsymbol{w}_{1}\right)+\sigma^{2}\right) \geq \gamma_{2} .
\end{aligned}
$$

This is a nonconvex problem, but the beamforming vectors that solve it can be parameterized as [7]

$$
\boldsymbol{w}_{i}=x_{i} \frac{\boldsymbol{\Pi}_{\boldsymbol{h}_{i i}} \boldsymbol{h}_{i j}}{\left\|\boldsymbol{\Pi}_{\boldsymbol{h}_{i i}} \boldsymbol{h}_{i j}\right\|}+y_{i} \frac{\boldsymbol{\Pi}_{\boldsymbol{h}_{i i}}^{\perp} \boldsymbol{h}_{i j}}{\left\|\boldsymbol{\Pi}_{\boldsymbol{h}_{i i}}^{\perp} \boldsymbol{h}_{i j}\right\|}
$$

for $i, j=1,2$ and $j \neq i$, where $\left(x_{i}, y_{i}\right) \in \mathcal{Q}$. Inserting (38) in (33)-(37) yields the equivalent optimization problem

$$
\begin{array}{ll}
\underset{t \in \mathbb{R}_{+},\left(x_{i}, y_{i}\right) \in \mathcal{Q}, i=1,2}{\operatorname{maximize}} & t \\
\text { subject to } & g_{11} x_{1} \geq \sqrt{\gamma_{1}^{\star} \sigma^{2}} \\
& \beta_{1} x_{1}+\tilde{\beta}_{1} y_{1} \geq \sqrt{\gamma_{1}^{\star}\left(g_{22}^{2} x_{2}^{2}+\sigma^{2}\right)} \\
& g_{22} x_{2} \geq \sigma t \\
& \beta_{2} x_{2}+\tilde{\beta}_{2} y_{2} \geq t \sqrt{g_{11}^{2} x_{1}^{2}+\sigma^{2}} .
\end{array}
$$

The coefficients in (39)-(43) are defined in Tab. I. The objective function (39) and the constraints (40) and (42) are linear in the optimization variables. The set $\mathcal{Q}$ is convex. Constraint (41) defines a SOC. However, (43) is a SOC constraint parameterized by $t$. So, we solve (39)-(43) by bisection over $t$ [8, Ch. 4]. An upper bound, $U$, on $t$ is obtained by setting $\gamma_{1}^{\star}=0$ and solving (39)-(43). Then, we have $x_{1}^{\star}=y_{1}^{\star}=0$ and (43) is a linear constraint. We set the lower bound to $L=0$ and set $t:=(U+L) / 2$. For $t$, we solve the following convex SOC feasibility problem.

$$
\begin{array}{ll}
\underset{\left(x_{i}, y_{i}\right) \in \mathcal{Q}, i=1,2}{\text { find }} & \left(x_{1}, y_{1}, x_{2}, y_{2}\right) \\
\text { subject to } & g_{11} x_{1} \geq \sqrt{\gamma_{1}^{\star} \sigma^{2}}, \\
& \beta_{1} x_{1}+\tilde{\beta}_{1} y_{1} \geq \sqrt{\gamma_{1}^{\star}\left(g_{22}^{2} x_{2}^{2}+\sigma^{2}\right)}, \\
& g_{22} x_{2} \geq \sigma t, \\
& \beta_{2} x_{2}+\tilde{\beta}_{2} y_{2} \geq t \sqrt{g_{11}^{2} x_{1}^{2}+\sigma^{2}} .
\end{array}
$$

If (44)-(48) is feasible we set $L:=t$, otherwise, we set $U:=t$. We iterate this procedure until convergence. Typically, a handful of iterations is needed. We get the optimal beamforming vectors $\left(\boldsymbol{w}_{1}^{\star}, \boldsymbol{w}_{2}^{\star}\right)$, by inserting the optimal solution $\left(x_{1}^{\star}, y_{1}^{\star}, x_{2}^{\star}, y_{2}^{\star}\right)$ into (38). In order to find the entire boundary, we let $\gamma_{1}^{\star}$ go from 0 to the maximum of $\min \left\{p_{1}\left(\boldsymbol{w}_{1}\right), q_{2}\left(\boldsymbol{w}_{1}\right)\right\} / \sigma^{2}$ for $\boldsymbol{w}_{1} \in \mathcal{W}$. Once the constants in Tab. I are computed, the complexity of (44)-(48) is constant with respect to the number of transmit antennas. In [7] it was proven that the TXs might not necessarily use the maximum available power at the Pareto boundary. However, it is easy to verify that at least one TX uses maximum power.

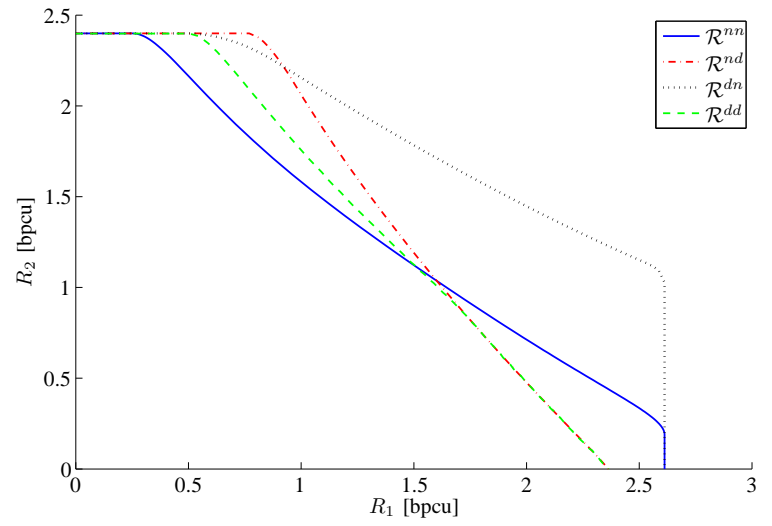

Fig. 1. Example of regions for $n_{T}=3$ and $\sigma^{2}=0.5$

\section{NumERICAL EXAMPLE AND CONCLUSION}

In Fig. 1, we illustrate the rate regions $\mathcal{R}^{n n}, \mathcal{R}^{d n}, \mathcal{R}^{n d}$, and $\mathcal{R}^{d d}$ for one realization of the channels, where $\boldsymbol{h}_{i j} \sim$ $\mathcal{C N}(\mathbf{0}, \boldsymbol{I}), i, j=1,2$. In this specific example, we see that $\mathcal{R}^{d n}$ constitutes almost the entire rate region $\mathcal{R}$. It is a typical result that the union of the two regions obtained when one RX decodes interference $\left(\mathcal{R}^{d n}\right.$ and $\mathcal{R}^{n d}$ ) is larger than the region $\mathcal{R}^{d d}$ obtained when both RXs decode interference. The reason for why $\mathcal{R}^{d d}$ is not the largest region is that in order to decode the interference we need extra power over the crosstalk channel. This comes at the cost of decreased power over the direct channel. So, when both RXs decode interference before decoding the desired signal, they might experience low power received from the direct channel. This implies low achievable rates.

In this paper we proposed an efficient method for finding the Pareto boundary of the rate region for the MISO IC with MUD capable receivers. The method is efficient in the sense that it has a complexity that is constant with respect to the number of transmit antennas. Also, the boundary can partly be found in closed form. The merit of the proposed method, compared to the previously known methods, is that it avoids the brute-force search over all feasible beamforming vectors.

\section{REFERENCES}

[1] A. Carleial, "Interference channels," IEEE Trans. Inf. Theory, Jan. 1978.

[2] E. G. Larsson and E. A. Jorswieck, "Competition versus cooperation on the MISO interference channel," IEEE J. Sel. Areas Commun., Sept. 2008.

[3] E. Karipidis and E. G. Larsson, "Efficient computation of the Pareto boundary of the MISO interference channel with perfect CSI," in Proc. WiOpt, May 2010.

[4] J. Lindblom, E. Karipidis, and E. G. Larsson, "Closed-form parameterization of the Pareto boundary for the two-user MISO interference channel," in Proc. IEEE ICASSP, May 2011.

[5] H. Sato, "The capacity of the Gaussian interference channel under strong interference," IEEE Trans. Inf. Theory, June 1981.

[6] A. S. Motahari and A. K. Khandani, "Capacity bounds for the Gaussian interference channel," IEEE Trans. Inf. Theory, Feb. 2009.

[7] K. M. Ho, D. Gesbert, E. Jorswieck, and R. Mochaourab, "Beamforming on the MISO interference channel with multi-user decoding capability," in Proc. IEEE Asilomar Conf., Nov 2010. Journal version submitted to IEEE Trans. Inf. Theory, 2011. Available: http://arxiv.org/abs/1107.0416.

[8] S. Boyd and L. Vandenberghe, Convex Optimization, Cambridge University Press, 2004. 